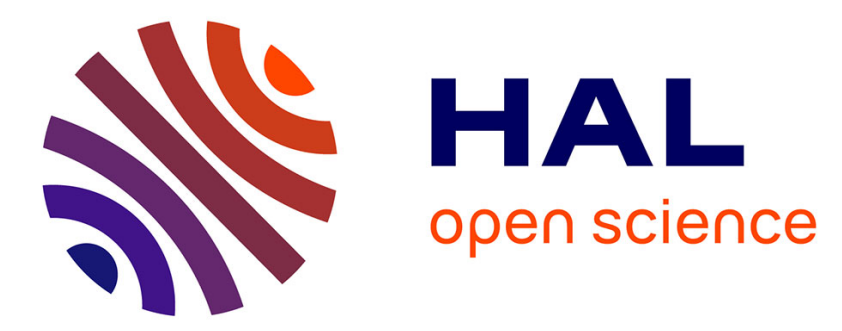

\title{
Temperature and rate dependent theory of plasticity of crystalline solids
}

\author{
P. Perzyna
}

\section{To cite this version:}

P. Perzyna. Temperature and rate dependent theory of plasticity of crystalline solids. Revue de Physique Appliquée, 1988, 23 (4), pp.445-459. 10.1051/rphysap:01988002304044500 . jpa-00245792

\section{HAL Id: jpa-00245792 https://hal.science/jpa-00245792}

Submitted on 1 Jan 1988

HAL is a multi-disciplinary open access archive for the deposit and dissemination of scientific research documents, whether they are published or not. The documents may come from teaching and research institutions in France or abroad, or from public or private research centers.
L'archive ouverte pluridisciplinaire HAL, est destinée au dépôt et à la diffusion de documents scientifiques de niveau recherche, publiés ou non, émanant des établissements d'enseignement et de recherche français ou étrangers, des laboratoires publics ou privés. 


\title{
Temperature and rate dependent theory of plasticity of crystalline solids
}

\author{
P. Perzyna \\ Institute for Fundamental Technological Research, Polish Academy of Sciences, \\ Swietokrzyska 21, 00- 049 Warsaw, Poland
}

(Reçu le 15 juin 1987, révisé le 11 janvier 1988 et accepté le 15 janvier 1988)

\begin{abstract}
The paper shows a new approach for formulation of temperature and rate dependent models of plastic behaviour of crystalline solids. It has sevcral particular objectives. The first is to sive a very brief overview of recent developments $0 i^{i}$ large strain constitutive laws for crystalline rate dependent plasticity. The second is to investigate how the main rechanisms on crystalline slip system can be incorporated in the general framework of continuum rate dependent large plastic deformation constitutive structure. The internal state variables have been precisely interpretec. Baseu on main mechanisms for crystalline slip system the evolution equations for the internal state variables introduced are proposed. The third objective is to illustrate how to include additional features such as work hardening, thermal softening etc. in constitutive structure proposea. Next objective is to focus attention on temperature dependence of plastic behaviour. The final goal is the discussion how these propositions can be extended to develop theories for large deformation, temperature and rate dependent, polycrystalline behaviour.
\end{abstract}

\section{Introduction}

The understanding of the physical origin and nature of the plastic behaviour of polycrystalline aggregates constitutes one of the major problems in modern materials science. Fundamental features of finite deformation, rate dependent jlastic flow of crystalline solids are discussed from microscopic and macroscopic phenomenolocical points of view. Particular models are proposed to nredict deformation textures and large strain, temperature and rate dependent and strain hardening behaviour of polycrystals from the known behaviour of single crystals . The posibility of making such a prediction rests on the tacit assumption that the mechanisms of plastic deformation in ac-gregates are substantially identical with those observed in single crystals.

constitutive relations for microelements are formulated on the basis of slip-induced. rate dependent plastic deformation. This model has been developed witilin the framcwork of a internal state variable material structure. The shearings and the critical shear stresses on slip system are introduced as internal state variables. This set of internal state variables is sucrested by nhysical mechanisms of rate dependent plastic flow on slip system considered.

Physical mechanisms of rate dependent ki- netic laws for shearinc are proposed.Attention is focused on physical mechanisms and models as follows:

(i) Creep mechanism of polycrystalline solids (cf. Stein and Low [72], Ashby and Frost [6], Hutchinson [29], Pan and Rice[51], Peirce, Asaro and Needleman [61], and Asaro and Teedleman [4]) .

(ii) Dislocation-point defect interaction mechanism (cf.Gilman [15,16]).

(iii) Viscoplastic flow model (cf.Bingham [7], Hohenemser and Prager [27], Prager[62], Sokolovski[71], Malvern [43] and Perzyna[5?-54]). (iv) Thermally activated mechanism (cf. Seecer $[67,68])$

(v) Phonon viscosity damping mechanism (cf. Mason $[47,48])$.

(vi) Phenomenological viscoplastic model for slip system (cf.Perzyna and Pęcherski [5y]).

(vii) Interaction of the thermally activated and phonon viscosity damping mechanism (cf. Kunar and Kumble[38] and Teodosiu and sidorofi [74]).

Experimental justifications for each of the particular mechanisms have been given and the range of applications has been discussed.

Particular attention is devoted to the phrsical interpretation of the transition from the ratc remendent nlastic flow to the 
inviscid plastic response. Rate incie zencent plastic response of a material has been considered as a limit case of the rate dependent kinetic laws for shearing proposed. Work hardening effects are carefully discussed and particular descriptions are riven. Influence of thermomechanical coupling is scrupulously investigated.

Rate type formulation of the temperature and rate dependent plastic behaviour of cry-* stalline solids is also presented.Comparison with internal state variable structure is jiven. Important result has been achieved Erom the investication of the conditions under which internal state variable and rate type material structures are isomorphic,i.e. describe the same material. It has been shown that the equivalence relation depends on the evolution equations postulated for the internal state variables as well as on the flux of the Kirchhoff stress tensor assuned in the rate type formulation. Thus, this similarity condition gives the physica? criterion on the definition of flux.

Particular examples are considered and liscussed.

2. Internal state variable constitutive model

2.1. Kinematics of finite deformations. In recent years it has been a great activity in cieveloping a general theory for the mechanics of finite elastic-plastic deformation of crystalline materials. This research was initiated by the pioneering work of Taylor [73].

Development of Taylor's conception was given by many researchers, cf. Argon [1], Asaro [2,3], Asaro and Needleman [4], Asaro and Pice [5], Bishop and Hill [8,9], Bui, Zaoui and Zarka [11], Budiansky and Wu[10], Havner [19], Havner and Shalaby [20], Hill $[21,22,23,24]$, Hill and Havner [25], Hill and Rice [26], Hutchinson [28,29], Iwakuma and Nemat-Nasser [30], Kocks [31,32], Kroner [34-36], Mandel [44], Milstein and Hill[46], Peirce, Asaro and Needleman $[60,61]$, Rice $[64,65,66]$ and Zarka [76]

is fundamental idea of this theory is that naterial flows through the crystal lattice n means of dislocation motion, whereas the lattice itself undergoes elastic deformations. The total deformation and rotation

of the crystal when it is under load is $\mathrm{gi-}$ ven jy the deformation gradient $F$.

As it has been postulated by Rĩce $[65,66]$ and Asaro and Rice [5] the deformation crádient can be written as (cf. also with the postulate introduced by tee [39]).

$$
\underset{\sim}{F}=\underset{\sim}{F^{*}} \cdot \underset{\sim}{F^{P}} \text {, }
$$

where $F^{*}$ is the lattice contribution to $\underset{\sim}{F}$ and is associated with stretching and rotation of the lattice, $\underset{\sim}{\mathrm{P}}$ is the deformation solely due to plastic shearing on crystallographic slip systems, see Fig.1. A particular slip system $\alpha$ is specified by the slip vectors $\underset{\sim}{s}(\alpha), \underset{\sim}{m}(\alpha)$, where $\underset{\sim}{s}(\alpha)$ gives the slip direction and $\underset{\sim}{m}(\alpha)$ is the slip plane normal. The vectors $\underset{\sim}{\underset{n}{s}}(\alpha)$ and $\underset{\sim}{m}(\alpha)$ are taken to be orthonormal in the undêforied lattice. As the crystal deforms the vectors $\underset{\sim}{s}(\alpha)$ and $\underset{\sim}{m}(\alpha)$ are stretched and rotated according to ${\underset{\sim}{*}}^{*}$. In the deformed lattice we have

$$
{\underset{\sim}{S}}^{*}(\alpha)=\underset{\sim}{F^{*}} \cdot \underset{\sim}{\underset{\sim}{S}}(\alpha), \quad \mathbb{\sim}^{*}(\alpha)=\underset{\sim}{m}(\alpha) \cdot \underset{\sim}{\mathbb{F}^{*}}{ }^{-1} \text {. }
$$

Since ${\underset{\sim}{S}}^{(\alpha)}$ and $\underset{\sim}{(\alpha)}$ are orthogonal so are $\mathrm{\sim}^{*}(\alpha)$ and $\mathrm{m}_{\sim}^{*}\left(\alpha \tilde{)}\right.$, i.e. $\underset{\sim}{(\alpha)} \cdot \sim_{\sim}^{\mathrm{m}}{ }^{(\alpha)}={\underset{\sim}{*}}^{*}(\alpha) \cdot{\underset{\sim}{m^{*}}}^{(\alpha)}=0$.

The deformation gradient remairing after unloading and upon returning the lattice to its orientation in the reference state is

$$
{\underset{\sim}{F}}^{*^{-1}} \cdot \underset{\sim}{\mathrm{F}}=\underset{\sim}{\mathrm{F}} \mathrm{P} \text {. }
$$

The plastic part of Eulerian velocity gradient in the current state $\underset{\sim}{\stackrel{\mathrm{F}}{F^{-1}}}$ is given by

$$
\begin{aligned}
& \underset{\sim}{\dot{F}} \cdot \underset{\sim}{F}{ }^{-1}-\underset{\sim}{\dot{F}}{ }_{\sim}^{F} *^{*-1}=\underset{\sim}{F} * \cdot \underset{\sim}{\dot{F}} P \cdot \underset{\sim}{P} P^{P^{-1}} \cdot \underset{\sim}{F}{ }^{*-1}= \\
& =\sum_{\alpha=1}^{\mathrm{n}}{\underset{\sim}{\mathrm{s}}}^{*}(\alpha){\underset{\sim}{\mathrm{m}^{*}}}(\alpha) \underset{\gamma}{\dot{\gamma}(\alpha)},
\end{aligned}
$$

where $\dot{\gamma}^{(\alpha)}$ is the rate of shearing on slip system $\alpha$.

By using relations (2.2) we have

$$
\begin{aligned}
& \underset{\sim}{\mathrm{F}} * \underset{\sim}{\stackrel{\mathrm{F}}{\mathrm{P}}} \cdot \underset{\sim}{\mathrm{F}^{\mathrm{P}^{-1}}} \cdot \underset{\sim}{\mathrm{F}^{*-1}}= \\
& =\underset{\sim}{F^{*}} \cdot \sum_{\alpha=1}^{\mathrm{n}} \underset{\sim}{\underset{\sim}{S}}(\alpha) \underset{\sim}{m}(\alpha) \underset{\gamma}{(\alpha)} \cdot \underset{\sim}{F^{*-1}} \text {. }
\end{aligned}
$$

The last result is equivalent to the expression for the rate $\underset{\sim}{\underset{P}{P}}$ given by Rice [65]

$$
\underset{\sim}{\dot{\vec{F}}} \mathrm{p}=\sum_{\alpha=1}^{\mathrm{n}} \underset{\sim}{\mathrm{s}}(\alpha) \underset{\sim}{\mathrm{m}}(\alpha) \underset{\gamma}{(\alpha)} \cdot \underset{\sim}{\mathrm{F}} \mathrm{p} .
$$

For each slip system, one can define the symmetric and anti-symmetric tensors

$$
\begin{aligned}
& \underset{\sim}{M}(\alpha)=\frac{1}{2}\left[{\underset{\sim}{*}}^{*}(\alpha) \underset{\sim}{\mathbb{m}^{*}}(\alpha)+\underset{\sim}{\operatorname{m}}{ }^{*}(\alpha) \underset{\sim}{\mathbb{S}^{*}(\alpha)}\right], \\
& \underset{\sim}{W}(\alpha)=\frac{1}{2}\left[{\underset{\sim}{s}}^{*}(\alpha) \underset{\sim}{m^{*}}(\alpha)-m_{\sim}^{*}(\alpha) \underset{\sim}{S^{*}}(\alpha)\right] \text {. }
\end{aligned}
$$

Then, instead of

$$
\underset{\sim}{\dot{F}} \cdot \underset{\sim}{F^{-1}}=\underset{\sim}{\dot{F}} * \cdot \underset{\sim}{F} *^{-1}+\underset{\sim}{F} * \cdot \underset{\sim}{\dot{F}} P \cdot \underset{\sim}{F} P^{-1} \cdot \underset{\sim}{F} *^{-1}
$$

we can write

$$
\underset{\sim}{\mathrm{D}}+\underset{\sim}{\Omega}=\mathrm{D}^{*}+\underset{\sim}{\Omega} *+\underset{\sim}{\mathrm{D}^{\mathrm{P}}}+\underset{\sim}{\Omega} \mathrm{P},
$$

where $\mathrm{D}$ is the symmetric rate of stretching tẽnsor and $\Omega$ is the anti-symmetric spin rate. The elãstic rates of stret ching and spin $D^{*}$ and $\Omega^{*}$ are.the symmetric and anti-symmétric parts of $\dot{F}^{*} \cdot F^{*}-1$, respectively, and the plastic parts of the rate of stretching and spin rate are determined by the relations

$$
\stackrel{\sim}{\mathrm{D}}^{\mathrm{P}}=\sum_{\alpha=1}^{\mathrm{n}} \dot{\gamma}^{(\alpha)} \underset{\sim}{\mathrm{M}}(\alpha),{\underset{\sim}{\Omega}}^{\mathrm{P}}=\sum_{\alpha=1}^{\mathrm{n}} \dot{\gamma}^{(\alpha)} \underset{\sim}{\mathrm{W}}(\alpha) \cdot(2 \cdot 10)
$$

2.2. Assumptions for crystalline solids. It has been assumed that the crystal elasticity is unaffected by slip and characterized by a strain energy function $\phi$. The rate of stress working per unit reference volume is 


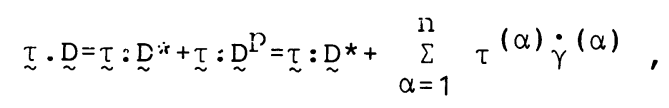

where $\underset{\tau}{\tau}$ is the Kirchhoff stress tensor, det $(\underset{\sim}{F})$ times the Cauchy stress, and

$$
\tau(\alpha)=\underset{\sim}{\tau: \underset{\sim}{M}}(\alpha)
$$

is the Schmid resolved shear stress on slip system $\alpha$.

Let $\mathrm{E}^{*}$ is the lattice Green strain, i.e.

then

$$
\underset{\sim}{E^{*}}=\underset{\sim}{\stackrel{r}{2}}\left(\underset{\sim}{F}{ }^{T} \cdot \underset{\sim}{F} *-\underset{\sim}{I}\right),
$$

$$
\begin{aligned}
& \tau: \underset{\sim}{\mathrm{D}}=\left[\underset{\sim}{\mathrm{F}^{*}}-1 \cdot \underset{\sim}{\tau} \cdot \underset{\sim}{\mathrm{F}^{*}}-\mathrm{T}\right]:{\underset{\sim}{\dot{E}}}^{*}+\sum_{\alpha=1}^{\mathrm{n}} \tau(\alpha) \underset{\gamma}{\dot{\gamma}}(\alpha) \\
& =\frac{\partial \phi}{\partial \underset{\sim}{E^{\star}}}: \dot{\sim}^{*}+\sum_{\alpha=1}^{n} \tau(\alpha) \underset{\gamma}{(\alpha)} \text {, }
\end{aligned}
$$

where ( ) ${ }^{-T}$ denotes the inverse transpose. i..ence

$$
\tau={\underset{\sim}{F}}^{*} \cdot \frac{\partial \phi}{\partial \underset{\sim}{E^{*}}} \cdot \underset{\sim}{F^{*}} \text { or } \underset{\sim}{\tau}=\underset{\sim}{F} * \underset{\sim}{T} \cdot \underset{\sim}{F}{ }^{T},
$$

if $\underset{\sim}{\mathrm{T}}$ denotes the second Piola-Kirchhoff stre ss tensor.

We can also write

$$
\begin{aligned}
\underset{\sim}{\tau}: \underset{\sim}{\mathrm{D}} & =\underset{\sim}{\tau}:\left({\underset{\sim}{\mathrm{F}}}^{*} \cdot{\underset{\sim}{\mathrm{F}}}^{*-1}\right)+\sum_{\alpha=1}^{\mathrm{n}} \tau(\alpha) \underset{\gamma}{\gamma}(\alpha) \\
& ={\underset{\sim}{\mathrm{T}}}_{\mathrm{R}}: \dot{\sim}^{\mathrm{F}}+\sum_{\alpha=1}^{\mathrm{n}} \tau(\alpha) \underset{\gamma}{ }(\alpha),
\end{aligned}
$$

where

$$
\underset{\sim}{\mathrm{T}} \mathrm{R}=\underset{\sim}{\tau} \cdot\left({\underset{\sim}{F}}^{*-1}\right)^{\mathrm{T}}
$$

denotes the first Piola-Kirchhoff stress tensor

2.3. Constitutive structure for crystal. It is worthy to comment here on the nature of rate dependent response of solids. It is well known fact that in many practical problems the actual behaviour of a material is governed by plastic as well as by rheologic effects. It can even be said that for many crystalline solids rheoloric effects are more pronounced after the plastic state is reached.

Recent research concerning the description of dynamic properties of crystals has shown that the application of the theory oi plasticity, in which the fundamental assur.:ption is that of rate independence leads to too large discrepancies between the theore tical and experimental results.

Thus there is no need to point out the advantages that can be gained by simultane. ous description of viscous and plastic effects. However, the difficulties of combined treatment of viscous and plastic phenomena are enormous. The viscous properties of the crystal introduce a time dependence of constitutive equations and the plastic properties, on the other hand, make these equations depend on the loading path. As a result of simultaneous handling of viscous and plastic properties, we obtain a dependence on time and the load history. A des- cription of strain in viscoplasticity will therefore involve the history of the crystal, expressed in the type of the loading process and the time. Different results will be obtained for different loading, paths and different time duration of the process considered.

Incorporation of material rate dependence into the constitutive formulation for crystalline solids is important not only for treatment of dynamical processes when rate sensitivity is large but even when the material response is only slightly rate sensitive because the rate independent formulation precludes an analysis of large strain plastic flow for a full range of material properties (cf.Needleman et al. [50]).

It is also deserving to remark on the general constitutive structures which can be used to the description of rate dependent plastic properties of crystals. We can choose between two of them, namely an internal state variable constitutive structure and a rate type structure. Both of them are widely used in the theory of plasticity.

Let us introduce a notion of the local configuration of a particle $x$ of the crystal at time $t$ as

$$
P(t)=F_{\sim}^{*}(t),
$$

where $\mathrm{F}^{*}$ is the lattice deformation gradient, and the local response of a particle $x$ at time $t$ as

$$
\mathrm{Z}(\mathrm{t})={\underset{\sim}{\mathrm{T}}}_{\mathrm{R}}(\mathrm{t}),
$$

where ${\underset{\sim}{R}}_{R}(t)$ is the first Piola-Kirchhoff stress tensor.

The intrinsic state $\sigma$ of a particle $x$ at time $t$ consists of a pair

$$
\sigma=(P(t), A(t)),
$$

i.e. the configuration $P(t)$ and the method of preparation $A(t)$.

The method of preparation aims at the description of the internal rearrangement of the material sample. In other words it determines the internal dissipation of a material of the crystal.

It is assumed that the initial-value problem

$$
\dot{A}(t)=\hat{A}(\sigma), \quad A(0)=A_{0}
$$

has a unique solution in the form

$$
A(t)=F\left(A_{0},{\underset{\sim}{F}}_{[0, t]}^{*}\right),
$$

where $F$ is called the solution functiona? and $\underset{\sim}{F}[0, t]$ denotes a segment of the lattice deformation gradient process.

The evolution of the intrinsic state is determined by the evolution function

$$
\begin{gathered}
\sigma=\hat{e}\left(\sigma _ { O ^ { \prime } } { \underset { \sim } { F } [ 0 , t ] ) } ^ { * } \left(F^{*}(t), F\left(A_{O},{\underset{\sim}{F}[0, t]}_{[0}^{*}\right) .\right.\right.
\end{gathered}
$$

The principle of determinism for the ma.. terial structure with internal state variai. les is expressed by the constitutive equation

$$
\underset{\sim}{T}(t)=\underset{\sim}{\hat{T}}(\sigma)=\hat{\sim}_{\sim}^{T}(\underset{\sim}{F} *(t), A(t)) .
$$


The function $\hat{\mathbb{T}}$ represents the response function for the first Piola-Kirchhoff stress tensor.

As a set of the internal state variables it is assumed

$$
A(t)=\left(\gamma^{(\alpha)}(t), g^{(\alpha)}(t)\right),
$$

where $\gamma^{(\alpha)}$ denote the shearings and $r$, $\alpha$ : are the critical stresses in the slip system $\alpha(\alpha=1,2, \ldots, n)$ of the crystal.

$(\alpha)$ The evolution equations for the shearings $\gamma^{(\alpha)}$ and for the critical
are postulated as follows

$$
\begin{aligned}
& \dot{\gamma}^{(\alpha)}(t)=\Gamma^{(\alpha)}(\sigma), \\
& \dot{g}^{(\alpha)}(t)=G^{(\alpha)}(\sigma),
\end{aligned}
$$

and the initial-values

$$
\gamma^{(\alpha)}(0)=\gamma_{0}^{(\alpha)}, g^{(\alpha)}(0)=g_{0}^{(\alpha)} \text {. }
$$

we evolution eruations (2.26) togetiner with the initial-values (2.27) define the fundamental kinetic laws for rate dependent plastic behaviour of the crystal.

For this particular choice of the internal state variables the evolution function is given by

$$
\begin{aligned}
& \sigma=\hat{e}\left(\sigma_{0}, \underset{\sim}{F}[0, t]\right) \\
& =\left(\underset{\sim}{F}(t), F\left(\gamma_{0}^{(\alpha)}, g_{0}^{(\alpha)},{\underset{\sim}{F}}_{[0, t]}^{*}\right)\right) \text {, }
\end{aligned}
$$

where $F$ denotes nov the solution functional of the initial-value problem (2.26) $(2.27)$.

The first Piola-Kirchhoff stress can be written in the form

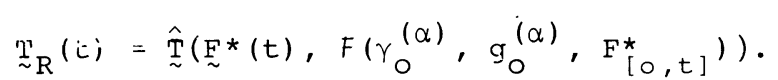

2.4. IJonisothermal process. Let us assume uniform distribution of temperature in the crystal. This assumption simplifies considerations and allows to focus our attention only on the internal dissipation effects.

The intrinsic state $\sigma$ is again a pair

$$
\sigma=(P(t), A(t) ;
$$

but the local configuration of a farticle $x$ at time $t$ is given by the Green strain tensor $\underset{\sim}{E^{*}}(t)$ and temperature $\theta(t), i . e$.

$$
P(t)=\left(\mathbb{\sim}^{*}(t), \theta(t)\right) .
$$

Iset us postulatc arain that a set of the internal state variables is represented by

$$
A(t)=\left(\gamma^{(\alpha)}(t), S^{(\alpha)}(t)\right)
$$

The local response of a particle $\mathrm{X}$ at time $t$ is now given by

$$
z(t)=(\Psi(t), N(t), \underset{\sim}{T}(t)),
$$

where $\Psi$ is the free energy, $N$ the eniropy and $\underset{\sim}{T}$ is the second Piola-Kirchhoff stress tẽnsor.

The principle of determinism is expressed by the relation

$$
\begin{gathered}
Z(t)=\hat{S}(\sigma) \\
=\hat{S}\left(\underset{\sim}{E^{*}(t),}, \theta(t), \gamma^{(\alpha)}(t), g^{(\alpha)}(t)\right),
\end{gathered}
$$

where

$$
\hat{S} \equiv\{\hat{\Psi}, \hat{N}, \underset{\sim}{\hat{T}}\}
$$

represents the free entrgy response iunction, the entropy response function and the stress response function.

The evolution equations are assumed in the form

$$
\dot{A}(t)=\hat{A}(\sigma), \quad A(0)=A_{O^{\prime}}
$$

i.e.

$\dot{\gamma}^{(\alpha)}(t)=\Gamma^{(\alpha)}(\sigma), \gamma^{(\alpha)}(0)=\gamma_{0}^{(\alpha)}$,

$\dot{\Phi}^{(\alpha)}(t)=G^{(\alpha)}(\sigma), \quad g^{(\alpha)}(0)=g_{0}^{(\alpha)}$.

The evolution function $\hat{e}$ is now given by

$$
\begin{aligned}
& \left.\sigma=\hat{e}\left(\sigma_{0}, P_{[0} t\right]\right) \\
& =\left({\underset{\sim}{E}}^{*}(t), \theta(t), F\left(\gamma_{0}^{(\alpha)}, S_{0}^{(\alpha)},{\underset{\sim}{E}[0, t]}^{E_{0}^{*}}, \theta_{[0, t]}\right)\right) \text {. }
\end{aligned}
$$

and it determines the evolution of the intrinsic state.

Thermodynamic restrictions superimposed on the constitutive relations (2.34) and the evolution equations $(2.37)$ give the results as follows

$$
\begin{aligned}
& \underset{\sim}{\mathrm{T}}(t)=2 \rho_{\mathrm{R}}{\underset{\sim}{\mathrm{E}^{*}}(t)}_{\hat{\Psi}, \quad \mathrm{N}^{\dot{*}}(t)=-\partial \theta(t)} \hat{\psi},(2.39) \\
& \hat{i}(\sigma)=-\frac{1}{\theta(t)}\left\{\sum_{\alpha=1}^{n} \partial \gamma(\alpha)(t) \hat{\Psi}^{(\alpha)}(t)\right. \\
& \left.+\sum_{\alpha=1}^{n} \partial^{(\alpha)}(t) \hat{\Psi}^{(\alpha)}(t)\right\} \geqq 0 \text {. }
\end{aligned}
$$


The last result is called the internal dissipation inequality, while the first two express the criterion of the selection of the response functions.

Choosing the free energy response function $\hat{\psi}$ such that the internal dissipation inequality (2.39) is satisfied at every instant of time ${ }_{t} \in\left[0, a_{p}\right]$ the response stress function $\underset{\sim}{\hat{T}}$ and the response entropy function $\hat{N}$ are uniquely determined by the relations $\tilde{(2.39)}$ and $(2.39)$.

Previous considerations from Section

2.2 suggest the postulate

$$
\partial_{\gamma}(\alpha) \hat{\Psi}=-\tau(\alpha) \text {. }
$$
form

If the hardening law is assumed in the

$$
\underset{\text { where }}{\quad \dot{g}^{(\alpha)}=\sum_{\alpha=1}^{n} \gamma^{(\alpha)}} \mathrm{h}_{\alpha \beta=1}(\gamma, \theta) \dot{\gamma}^{(\beta)},
$$

is taken to be the sum of the slip on all slip systems at the current instant, then the rate of internal dissipation is defined by

$$
\begin{aligned}
& \hat{i}(\sigma)=\frac{1}{\theta(t)} \sum_{\alpha=1}^{n}\{\tau(\alpha) \dot{\gamma}(\alpha) \\
& \left.-\partial_{g}(\alpha)^{\hat{\psi}} \sum_{\beta=1}^{n} h_{\alpha \beta}(\gamma, \theta) \dot{\gamma}(\beta)\right\} .
\end{aligned}
$$

Taking advantage of the relation

$$
\tau(\alpha)=\underset{\sim}{M}(\alpha):\left(\underset{\sim}{F} \cdot \underset{\sim}{T} \cdot \underset{\sim}{T}{ }^{T}\right)
$$

we can write

$$
\begin{aligned}
& \hat{i}(\sigma)=\frac{1}{\theta(t)} \sum_{\alpha=1}^{\mathrm{n}}\{\underset{\sim}{M}(\alpha): \underset{\sim}{\mathrm{F}} \cdot \underset{\sim}{\mathrm{T}} \cdot \underset{\sim}{\mathrm{F}} \mathrm{T}) \dot{\gamma}(\alpha) \\
& \left.-\partial_{\mathrm{g}}(\alpha)^{\hat{\Psi}} \sum_{\beta=1}^{\mathrm{n}} \mathrm{h}_{\alpha \beta}(\gamma, \theta) \dot{\gamma}(\alpha)\right\} .
\end{aligned}
$$

The second Piola-Kirchhoff stress tensor $\underset{\sim}{\mathrm{T}}$ is determined by the relation $(2.39)$, i.e.

$$
\underset{\sim}{T}(t)=\underset{\sim}{\hat{T}}\left({\underset{\sim}{*}}^{*}(t), \theta(t), F\left(\gamma_{0}^{(\alpha)}, g_{0}^{(\alpha)}, \underset{\sim}{E^{*}}[0, t], \theta[0, t]\right)\right) .
$$

The functional $F(\cdot)$ arising in the constitutive law (2.46) plays very important role in the rate and temperature dependent theory of plasticity of crystalline solids. It describes the pattern of internal rearrangement in crystal and it has been denoted by Rice [65] by Pir and by Hill and Rice [26].

2.5. Rate dependent kinetic laws. The rate and temperature dependence of the flow stress of metal crystals can be explained by different physical mechanisms of dislocation motion. The microscopic processes combine in various ways to give several groups of deformation mechanisms, each of which can be limited in its rate.

It is convenient to discuss each group in detail. (i) Dislocation creep mechanism. A pure empirical power relation between strain rate and stress is often used to characterize steady creep of metals and other materials at temperature above one-third of the melting point. At this range of temperature there is sufficient mobility of vacancies to allow dislocation to climb as well as glide (cf.Ashby and Frost [6]). Deformation is possible at a lower stress than would be needed for glide alone.

The connection between the shear rate and resolved shear stress on the $\alpha$ system for high temperature can be described by the semi-empirical equation (cf.Stein and Low [72], Ashby and Frost [6], Hutchinson [29], Pan and Rice [51], Peirce, Asaro and Needleman [61], and Asaro and Needleman [4])

$$
\dot{\gamma}^{(\alpha)}=\eta_{c}^{(\alpha)}\left[\frac{\tau^{(\alpha)}}{g_{C}^{(\alpha)}}\right]^{\frac{1}{m}} \operatorname{sgn} \tau^{(\alpha)},
$$

where $\eta_{C}(\alpha)$ is a convenient reference creep rate for the $\alpha$ system, and in general it is temperature dependent coefficient. The reference stress $g_{C}(\alpha)$ for the $\alpha$ system is a strong function of temperature. The exponent $\mathrm{m}$ also depends on temperature although somewhat less strongly.

Ashby and Frost [6] have broadly surveyed polycrystal and crystal data for many metals to determine the range of temperature and stress over which steady creep can be reasonably approximated by a power law stress dependence. The deformation map shown in Fig.2 is for pure nickel with a grain size of $10 \mu \mathrm{m}$. The assumption of steady-state flow is acceptable for dislocation creep mechanism.

(ii) Dislocation-point defect interaction mechanism. It has been proposed by Cilman [15, 16 ] that the velocity of dislocations in crystalline materials can be represented by

$$
v=v^{\infty} \exp (-g / \tau),
$$

where $v^{\infty}$ is the maximum velocity with which dislocations can move through the crystal lattice, $\tau$ is the resolved shear stress for the glide system and $g$ is a characteristic drag stress. Multiplying both sides of the relation $(2.48)$ by $\rho_{\mathrm{M}} \mathrm{b}$, where $\rho$ denotes the density of moving ${ }^{M}$ dislocations in the system $\alpha$ of the crystal and $\underset{\sim}{b}$ is the Burgers vector, we can obtain the Gilman's kinetic law for the $\alpha$ slip system

$$
\dot{\gamma}^{(\alpha)}=n_{G}^{(\alpha)}\left[\exp \left(-\frac{g_{G}^{(\alpha)}}{\tau}\right)\right] \text {, }
$$

where

$$
\dot{\gamma}^{(\alpha)}=\rho_{M} \text { bv and } n_{G}^{(\alpha)}=\rho_{M} b v^{\infty}
$$

have very well known physical interpretation. Experimental justification of Gilman relation was given by Gilman and Johnston[17].

(iii) Viscoplastic flow mechanism (model).It has been proposed that the rate dependent plastic flow is described by the relation (cf.Bingham [7], Hohenemser and Prager [27], Prager [62], Sokolovski [71], Malvern[43] and 


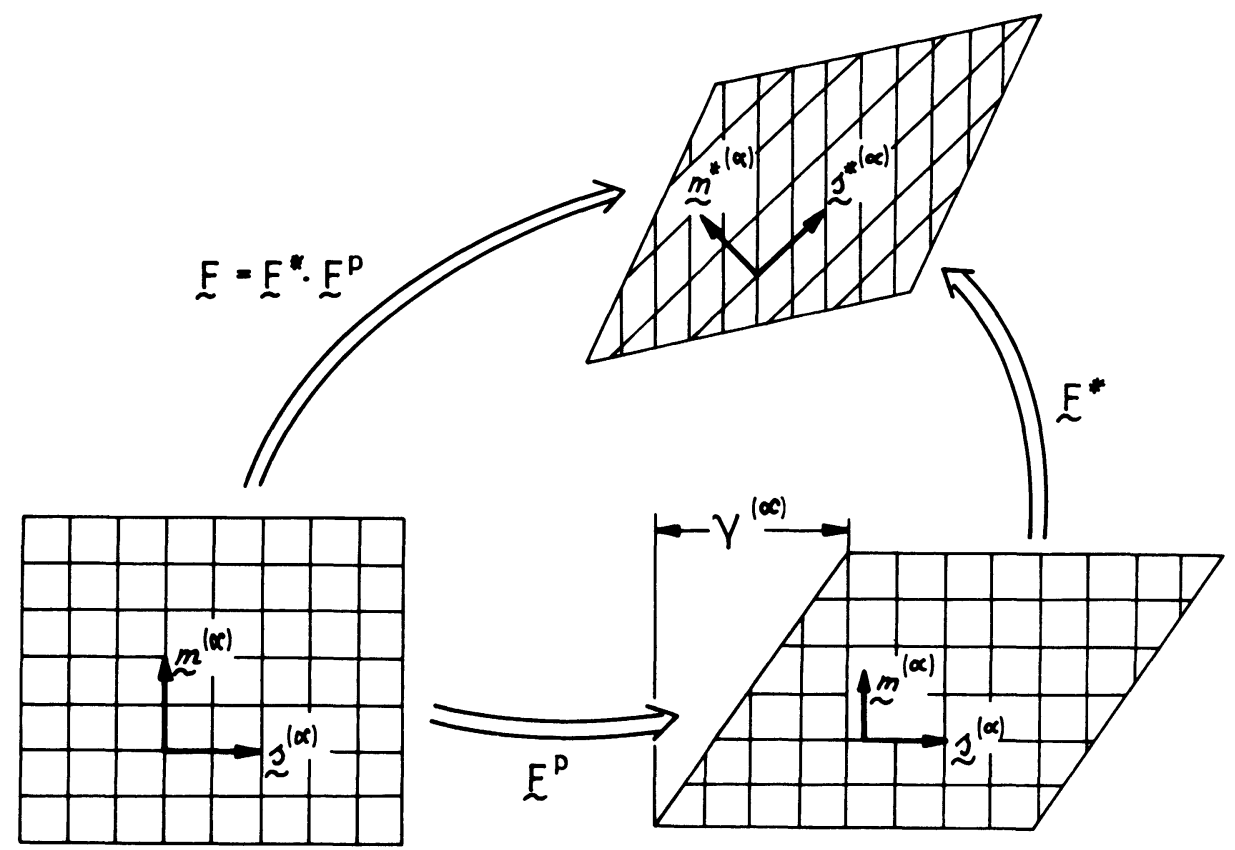

Fig.1. Decomposition of the deformation gradient

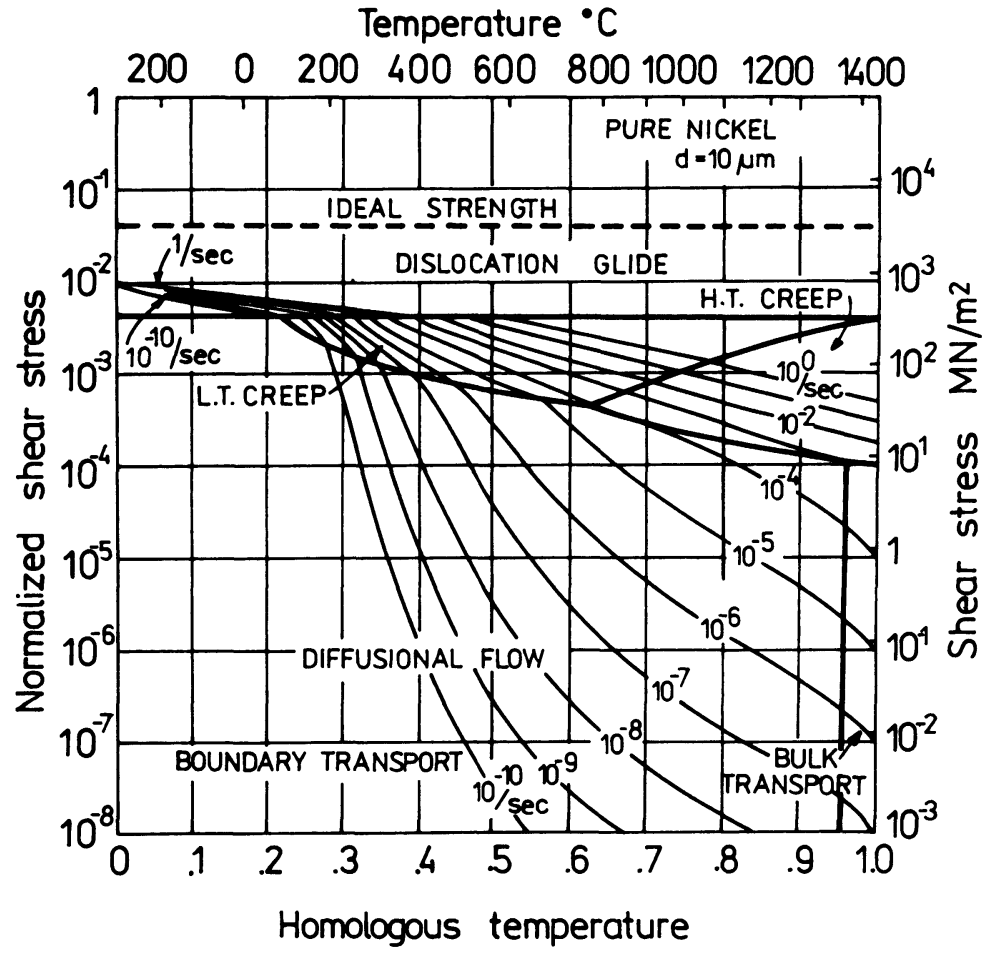

Fig.2. A map for nickel with a grain size of $10 \mu \mathrm{m}$ (After Ashby and Frost [6]) 
Perzyna $[52,53,54])$

$$
\dot{\gamma}^{(\alpha)}=\eta_{\mathrm{v}}^{(\alpha)}\left\langle\Phi\left[\frac{\tau^{(\alpha)}}{g_{\mathrm{v}}^{(\alpha)}}-1\right]>\operatorname{sgn} \tau^{(\alpha)},\right.
$$

where

$$
\langle[]\rangle=\left\{\begin{array}{lll}
0 & \text { if } \tau^{(\alpha)} \leqq g_{v}^{(\alpha)}, \\
{[\text { ] }} & \text { if } \tau^{(\alpha)}>g_{v}^{(\alpha)},
\end{array}\right.
$$

$\eta_{\mathrm{v}}^{(\alpha)}$ has dimension of rate of shearing and is assumed as a material viscosity coefficient for the slip system $\alpha, g_{\mathrm{v}}^{(\alpha)}$ has interpretation of the critical shear stress in the system $\alpha$ and a function $\Phi$ is determined basing on the available experimental results for particula $f_{\alpha}$ material $(\alpha)$ For nonisothermal processes $\eta_{\mathrm{v}}^{(\alpha)}$ and $\mathrm{g}_{\mathrm{v}}(\alpha)$ are assumed to depend on temperature.

In practical applications the power and exponential functions have been most frequently used, i.e.

$$
\begin{aligned}
& \Phi[\quad] \equiv\left[\frac{\tau^{(\alpha)}}{\mathrm{g}_{\mathrm{v}}^{(\alpha)}}-1\right]^{\mathrm{n}}, \mathrm{n}=1,3, \ldots \\
& \Phi[\quad] \equiv\left\{\exp \left[\frac{\tau^{(\alpha)}}{\mathrm{g}_{\mathrm{v}}^{(\alpha)}}-1\right]-1\right\} .
\end{aligned}
$$

Experimental justifications of the assumed postulates of the model and the discussion of its range of applicability have been Given Jy Lindholm [40,41,42], Campbell [12], Randal and Campbel1 [63] and recently by Shindoh [69]. In Fig. 3 the results for mild steel obtained under combined stress conditions by Shindoh [69] have been shown.

(iv) Thermally activated mechanism. It is now generally recognized that the plastic deformation of crystalline materials has dynamic nature and has been established as a thermally activated process dependent upon time, temperature, and strain rate. The evolution of the activation parameters is a widely used technique for the identification of the mechanisms controlling the rate of deformation and has been applied to b.c.c., f.c.c., h.c.p. metals, intermetallic compounds and ionic and ceramic crystals (cf.the review paper by Evans and Rawlings [14] and books by Nabarro[49] and Kocks, Argon, and Ashby [33]).

When a dislocation moves through a crystal lattice a force is exerted upon it by obstacles present in the lattice. This force can be separated into two components, a long-range force and a short-range force.

The stress to overcome short-range obstacles is temperature-dependent whereas that surmount fixed long-range obstacles generally depends upon temperature only through the temperature dependence of the shear modulus. For this reason the obstacles are often referred to as thermal and athermal, respectively. When both types of obstacles are present in a lattice the applied stress is usually comprised of both thermal and athermal components

$$
\tau=\tau^{\#}+\tau_{\mu},
$$

where $\tau^{\#}$ is the thermal (or effective) stress and $\tau_{\mu}$ is the athermal stress.

Plastic deformation occurs by the movement of a large number of dislocations through an array of obstacles.

At any finite temperature, coherent atomic fluctuations can assist the applied stress in moving a dislocation past obstacles.

The average velocity $v$ of a dislocation that surmounts obstacles with the assistance of thermal fluctuations is assumed to be an Arrhenius-type relationship

$$
\mathrm{v}=A \mathrm{~L}^{-1} \nu \exp \left(-\frac{\Delta \mathrm{G}}{\mathrm{k} \theta}\right)
$$

where $v$ is the frequency of vibration of the dislocation and $A L^{-1}$ is the distance moved after a successful fluctuation, and $\Delta G$ is the activation energy (Gibbs free energy), $k$ is the Boltzmann constant and $\theta$ is actual absolute temperature.

For the $\alpha$ slip system Eq. (2.56) can be written in the form

$$
\gamma^{(\alpha)}=\eta_{T}^{(\alpha)} \exp \left\{-\psi\left[\left(\tau^{(\alpha)}-g_{T}^{(\alpha)}\right) L b\right] / k \theta\right\},
$$

where $\quad \eta_{T}^{(\alpha)}=A L^{-1} \nu b \rho_{M}$,

$$
\Delta G=\psi\left[\left(\tau^{(\alpha)}-g_{T}^{(\alpha)}\right) L b\right]=\psi\left(\tau^{\#(\alpha)} L b\right),
$$

$$
g_{T}^{(\alpha)}=\tau_{\mu}^{(\alpha)}
$$

Seeger $[67,68]$ has proposed the linear form of the activation energy $\Delta G$, i.e.

$$
\Delta G=\frac{U_{0}^{(\alpha)}}{k \theta}-\left[\left(\tau^{(\alpha)}-g_{T}^{(\alpha)}\right) \frac{v^{*}}{k \theta}\right],
$$

where $U^{(\alpha)}$ denotes the constant value of the activation energy in the $\alpha$ slip system and $v^{*}$ is the activation volume.

Experimental justifications of the thermally activated mechanism and the discussion of the range of strain rate and temperature for particular materials have been given in many papers.

Particular importance for our purposes have results obtained by Campbell and Ferguson [13]. In Fig.4 the variation of lower yield stress of mild steel is shown. The same results are presented in Fig. 5 showing the variation of lower yield stress with temperature at constant strain rate. From Figs 4 and 5 we can directly determine the range of applicability of the thermally activated mechanism for plastic flow of mild steel and observe a very strong dependence of temperature. In Fig. 6 the effect of pre-straining is shown. This result has a great importance for the description of the work-hardening effect. Similar results have been obtained for polycrystalline aluminium by Hauser, Simmons and Dorn [18],cf.Fig.7.

(v) Damping mechanism (phonon viscosity). With increasing dislocation velocities at high enough stress or in perfect crystal 


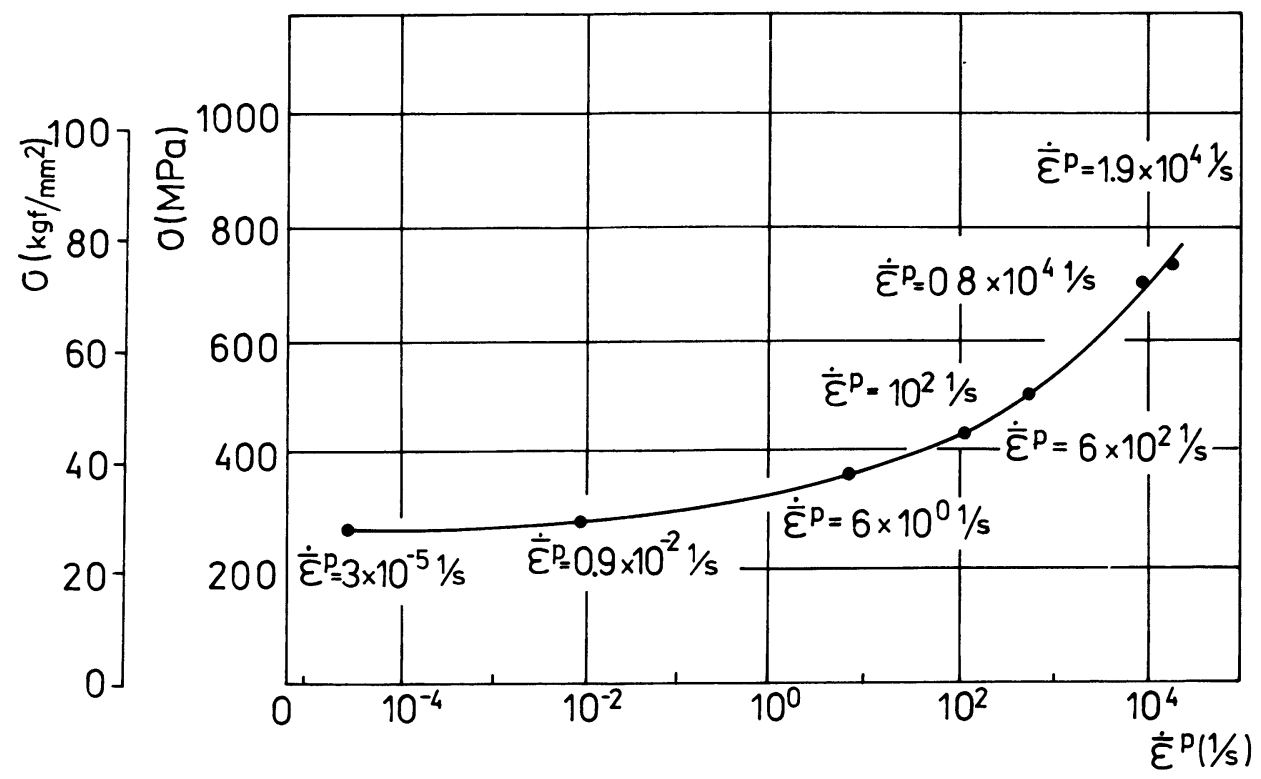

Fig.3. Variation of equivalent stress with equivalent strain rate for mild steel, at room temperature and constant equivalent strain (After Shindoh [69])

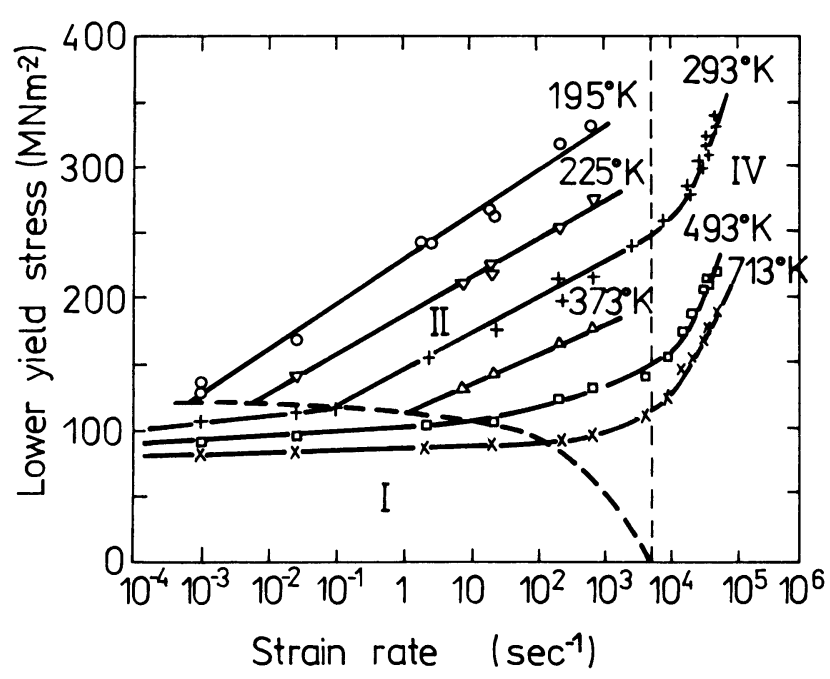

Fig.4. Variation of lower yield stress with strain rate for mild steel, at constant temperature (After Campbell and Ferguson [13])

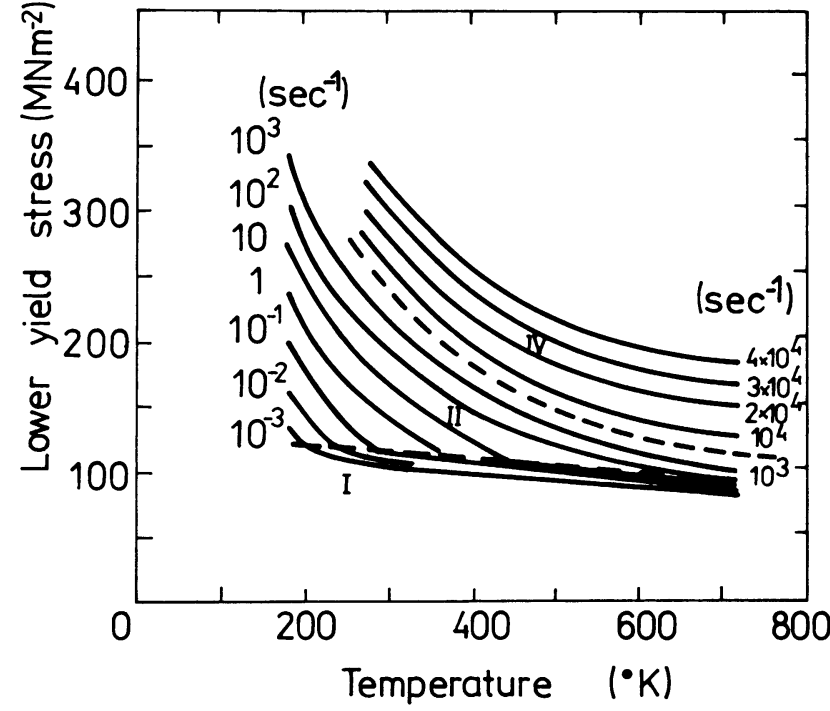

Fig.5. Variation of lower yield stress with temperature for mild steel, at constant strain rate (After Campbell and Ferguson [13]) 


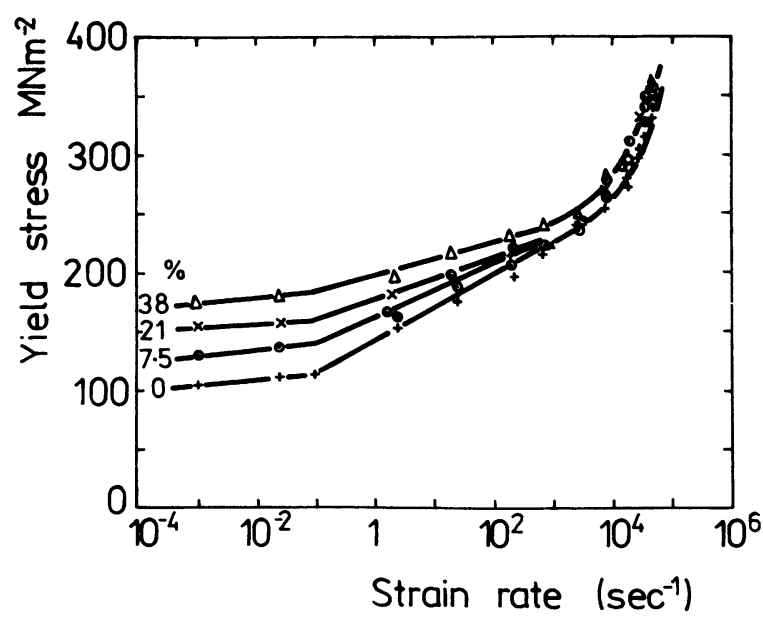

Fig.6. Effect of pre-straining for mild steel (After Campbell and Ferguson [13])

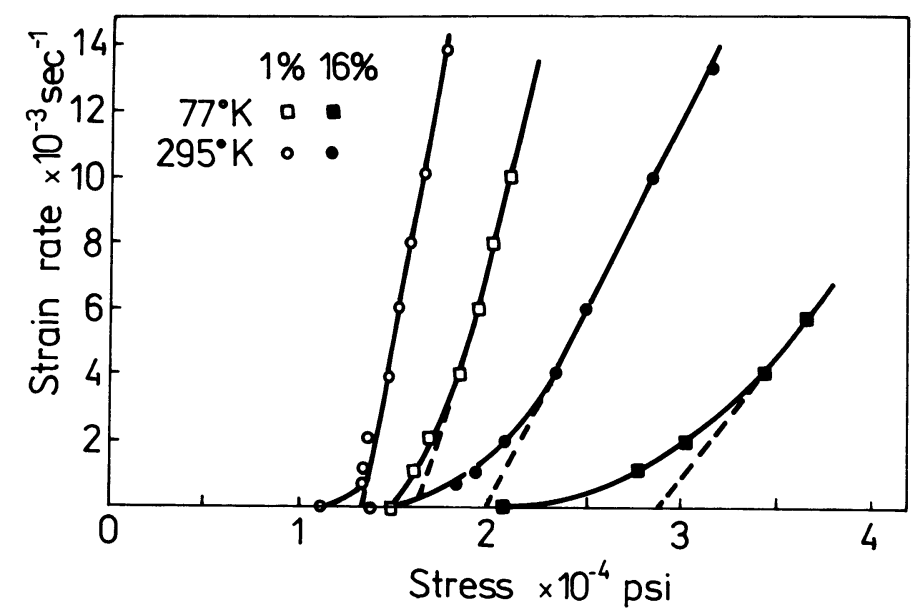

Fig.7. The strain rate dependence of the yield stress for polycrystalline aluminium. The data of Hauser, Simmon and Dorn [18] are plotted on a

linear scale

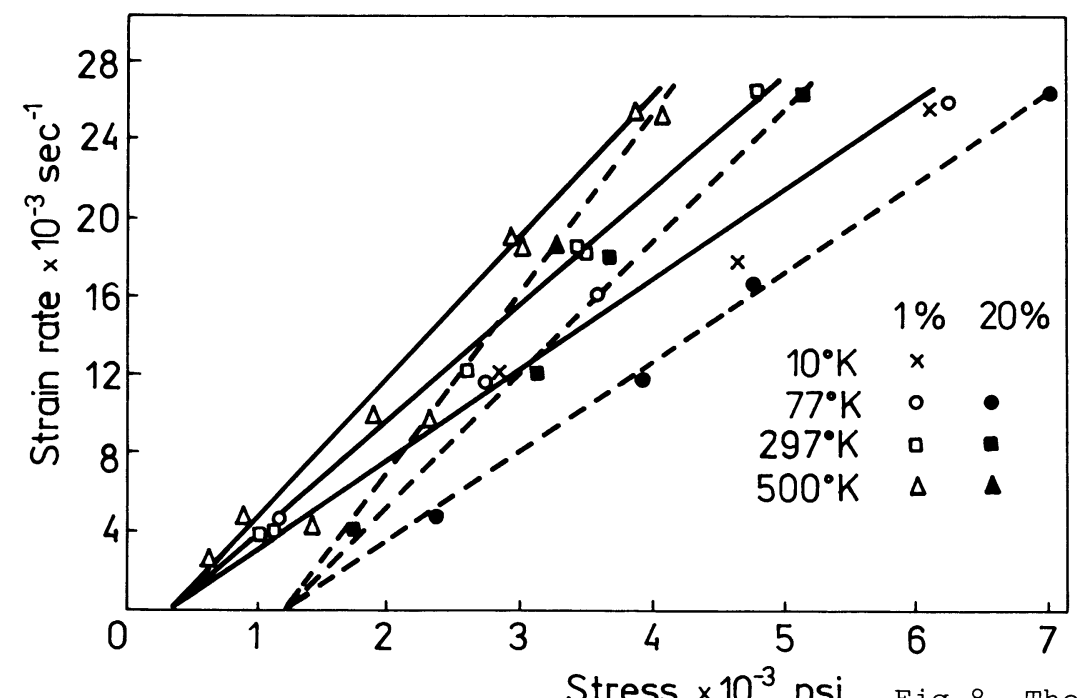

Stress $\times 10^{-3}$ psi Fig.8. The strain rate dependence of the flow stress of aluminium single crystals deformed by $(111)<1 \frac{110\rangle}{2}$ slip (After Kumar, Hauser and Dorn [37]) 
the velocity is only governed by the phonon damping mechanism. The phonon viscosity theory has been developed by Mason $[47,48]$ (c.f. Nabarro [49]). At very high strain rates the applied stress is high enough to overcome instantaneously the dislocation barriers without any aid fromthermal fluctuations This is true for the flow stress $\tau(\alpha)>g_{D}^{(\alpha)}$, where $g_{D}^{(\alpha)}$ is attributed to the stress needed to overcome the forest dislocation barriers to the dislocation motion and is called the back stress.

In this region the evolution equation for the plastic shearing has the form

$$
\dot{\gamma}^{(\alpha)}=\frac{\rho_{M} b^{2} g_{D}^{(\alpha)}}{B}\left[\frac{\tau^{(\alpha)}}{g_{D}^{(\alpha)}}-1\right] \operatorname{sgn} \tau^{(\alpha)},
$$

where $B$ is called the dislocation drag coefficient. If we introduce the denotation

$$
\eta_{D}^{(\alpha)}=\frac{\rho_{M} b^{2}}{B} \text {, }
$$

than the evolution equation $(2.60)$ takes the form

$$
\dot{\gamma}^{(\alpha)}=\eta_{D}^{(\alpha)}\left[\tau^{(\alpha)}-g_{D}^{(\alpha)}\right] \operatorname{sgn} \tau^{(\alpha)} .
$$

It is noteworthy that the dislocation drag coefficient $B$ can be interpreted as a generalized damping constant for phonon viscosity and phonon scattering mechanisms, i.e.

$$
\mathrm{B}=\mathrm{B}_{\mathrm{pv}}+\mathrm{B}_{\mathrm{ps}} \text {. }
$$

Experimental justification of the damping mechanism can be found in the paper by Kumar, Hauser and Dorn [37]. Fig.8 shows the strain rate dependence of the flow stress of aluminium single crystals deformed by (111) <1 10$\rangle$ slip.

The ratio $\mathrm{B} / \rho$ can be obtained from the slop of the linear portion of the shear stress against the shear strain rate curve, and from this ratio the value of $B$ can be calculated if a value of $\rho_{M}$ is assumed. (vi) Phenomenological viscoplastic model for slip system. Perzyna and Pecherski [59] suggested that viscoplastic strain is produced by the expansion of dislocation loops over the glide planes of active slip systems. They limited considerations to the region of moderate temperature in which the mechanisms of twinning, dislocation climb or diffusion are negligible.

The phenomenological model of plastic glide in the $\alpha$ slip system derived from the physical considerations leads to the following evolution equations

$$
\begin{aligned}
& \dot{\gamma}^{(\alpha)}=\frac{\eta_{\mathrm{P}}^{(\alpha)}}{1-\frac{\dot{\gamma}_{\mathrm{q}}^{(\alpha)}}{\dot{\gamma}^{(\alpha)}}}\left\langle\Phi\left[\mathrm{A}\left(\theta, \mathrm{g}_{\mathrm{P}}^{(\alpha)}\right)\left(\frac{\tau^{(\alpha)}}{\mathrm{g}_{\mathrm{P}}^{(\alpha)}}-1\right)\right]\right\rangle \operatorname{sgn} \tau^{(\alpha)}, \\
& \dot{\mathrm{g}}_{\mathrm{P}}^{(\alpha)}=\mathrm{h}\left(\theta, \gamma, \mathrm{g}_{\mathrm{P}}^{(\alpha)}\right) \dot{\gamma}^{(\alpha)},
\end{aligned}
$$

where $\dot{\gamma}^{(\alpha)}$ denotes the quasi static

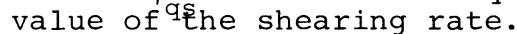

This model gives the uniform description of plastic deformation in an extensive range of strain rates and encompasses rate-sensitivity as well as rate indepndent behaviour of materials.

Experimental justification of the model has been discussed by Perzyna [58] and Perzyna and Pecherski [59].

(vii) Interaction of the thermally activated and phonon damping mechanisms (cf.Kumar and Kumble [38], Teodosiu and Sidoroff [74] and Perzyna [56]). If a dislocation is moving through the rows of barriers, then its velocity can be determined by the expression

$$
\mathrm{v}=A \mathrm{~L}^{-1} /\left(t_{\mathrm{S}}+\mathrm{t}_{\mathrm{B}}\right),
$$

where $A L^{-1}$ is the average distance of dislocation movement after each thermal activation, $t$ is the time a dislocation spent at the obstacle and $t_{B}$ is the time of travelling between the barriers.

The shearing rate in the $\alpha$ slip system is given by the relationship

$$
\begin{aligned}
\dot{\gamma}^{(\alpha)} & =\eta_{T}^{(\alpha)}<\exp \left\{\psi\left[\left(\tau^{(\alpha)}-g_{T}^{(\alpha)}\right) L b\right] / k \theta\right\} \\
& \left.+A B L^{-1} \nu /\left(\tau^{(\alpha)}-g_{D}^{(\alpha)}\right) \underset{\sim}{b}\right\rangle^{-1}
\end{aligned}
$$

where

$$
\begin{gathered}
\eta_{T}^{(\alpha)}=\rho_{M} b_{A L^{-1} \nu,} \\
\eta_{T}^{(\alpha)} \frac{b}{A B L^{-1} \nu}=\frac{\rho_{M} b^{2}}{B}=\eta_{D}^{(\alpha),}
\end{gathered}
$$

and two effective stresses

$\tau_{T}^{\#(\alpha)}=\tau^{(\alpha)}-g_{T}^{(\alpha)}$ and $\tau_{D}^{\#(\alpha)}=\tau^{(\alpha)}-g_{D}^{(\alpha)}$

are separately defined for the thermally activated and phonon damping mechanisms, respectively.

If the time $t_{B}$ taken by the dislocation to travel between the barriers in a viscous phonon medium is negligible when compared with the time $t_{S}$ spent at the obstacle, then

$$
\mathrm{v}=\frac{A \mathrm{~L}^{-1}}{\mathrm{t}_{\mathrm{S}}}
$$

and we can focus our attention on the analysis of the thermally activated process. When the ratio $t_{B} / t_{S}$ increases then the dislocation velocity $(2.65)$ can be approximated by the expression

$$
\mathrm{v}=\frac{A \mathrm{~L}^{-1}}{\mathrm{t}_{\mathrm{B}}}
$$

for the phonon damping mechanism.

Experimental justification has been given by Kumar and Kumble [38] and Shioiri, Satoh and Nishimura [70]. 
2.6. Rate independence. Let us discuss the assumptions which lead to rate independence of the kinetic laws introduced in section 2.5 .

(i) Dislocation creep mechanism. If the rate of shearing on the system $\alpha$ is

$$
\dot{\gamma}^{(\alpha)}=n_{c}^{(\alpha)},
$$

throughout the deformation history than the resolved shear stress $\tau(\alpha)$ on the system $\alpha$ is equal to $g_{c}(\alpha)$, i.e.

$$
\tau^{(\alpha)}=g^{(\alpha)} \text {. }
$$

This equation can be viewed as a reference $\tau-\gamma$ relation for each slip system. we can postulate $g_{c}^{(\alpha)}$ in the form

$g_{c}^{(\alpha)}=g_{0}^{(\alpha)}\left(1+h_{0} \gamma^{\frac{1}{\mu_{0}}}\right)\left[1-h_{1}\left(\frac{\theta-\theta_{0}}{\theta_{0}}\right)^{\frac{1}{\mu_{1}}}\right]$,

where

$$
\gamma=\sum_{\alpha=1}^{n} \gamma^{(\alpha)},
$$

and in this case $g_{C}^{(\alpha)}$ does not play the role of the internal state variables. In second case we can postulate that $g^{(\alpha)}$ are determined by the evolution equations

$$
g_{C}^{(\alpha)}=\sum_{\alpha=1}^{n} h_{\alpha \beta}(\theta, \gamma) \dot{\gamma}^{(\beta)},
$$

than

$$
g_{c}^{(\alpha)}=g_{0}^{(\alpha)}+\int \sum_{\alpha=1}^{n} h_{\alpha \beta}(\theta, \gamma) \dot{\gamma}^{(\beta)} d t,
$$

where the integral is, of course, path dependent.

(ii) Dislocation-point defect interaction mechanism. If

$$
\dot{\gamma}^{(\alpha)}=\eta_{G}^{(\alpha)},
$$

then

$$
\tau^{(\alpha)}=g_{G}^{(\alpha)},
$$

so, we have similar result as in previous case.

(iii) Viscoplastic flow model. Let the viscosity coefficients $\eta_{v}^{(\alpha)}$ tend to infinity, i.e.

and

$$
\eta_{\mathrm{v}}^{(\alpha)} \rightarrow \infty
$$

then

$$
\tau^{(\alpha)}=g_{v}^{(\alpha)} \text {, }
$$

is undetermined parameter. We can determine $\dot{\gamma}^{(\alpha)}$ from the work hardening law

$$
\dot{g}_{\mathrm{v}}^{(\alpha)}=\sum_{\alpha=1}^{\mathrm{n}} \mathrm{h}_{\alpha \beta}(\gamma, \theta) \dot{\gamma}^{(\beta)} \text {. }
$$

We have the inviscid plastic flow law

$$
\dot{\gamma}^{(\alpha)}=\sum_{\alpha=1}^{\mathrm{n}} \mathrm{h}_{\alpha \beta}^{-1}(\gamma, \theta) \dot{\tau}^{(\beta)} \text {. }
$$

If the work hardening law has the form

$$
\mathrm{g}_{\mathrm{v}}^{(\alpha)}=\psi\left(\gamma^{(\alpha)}\right),
$$

then we have the inviscid deformation plastic law

$$
\gamma^{(\alpha)}=\psi^{-1}\left(\tau^{(\alpha)}\right) \text {. }
$$

(iv) Thermally activated mechanism. Since

$$
\tau^{(\alpha)}=g_{T}^{(\alpha)}+\frac{1}{L b} \psi^{-1}\left[k \theta \ln \frac{n_{T}^{(\alpha)}}{\dot{\gamma}^{(\alpha)}}\right],
$$

if

$$
\dot{\gamma}^{(\alpha)} \rightarrow \dot{\eta}_{\mathrm{T}}^{(\alpha)} \text { then } \tau^{(\alpha)}=\mathrm{g}_{\mathrm{T}}^{(\alpha)} \text {. }
$$

(v) Phonon viscosity damping mechanism. Let

$$
\eta_{D}^{(\alpha)} \rightarrow \infty \quad \text { and } \tau^{(\alpha)}=g_{D}^{(\alpha)}
$$

then

$$
\dot{\gamma}^{(\alpha)}=\Lambda^{(\alpha)}
$$

and the result is similar as in the viscoplastic flow model.

(vi) Phenomenological viscoplastic model for slip system. For this case we have

$$
\tau^{(\alpha)}=g_{\mathrm{P}}^{(\alpha)}\left\{1+\Phi^{-1}\left[\frac{\dot{\gamma}^{(\alpha)}}{\eta_{\mathrm{P}}^{(\alpha)}}\left(1-\frac{\dot{\gamma}_{\mathrm{qs}}^{(\alpha)}}{\dot{\gamma}^{(\alpha)}}\right)\right]\right\} .
$$

If

$$
\dot{\gamma}^{(\alpha)} \rightarrow \dot{\gamma}_{\text {qs }}^{(\alpha)} \text { then } \tau^{(\alpha)}=g_{p}^{(\alpha)} \text {. }
$$

This result was obtained first by Perzyna [58] for polycrystalline solids.

2.7. Internal equilibrium. The condition

$$
\dot{A}(t)=0 \text { for } t \in\left[t_{1}, t_{2}\right] \subset\left[0, d_{P}\right]
$$

defines the internal equilibrium for the crystal.

$$
\text { It means that }
$$

$$
\mathrm{A}=\text { const. }
$$

for the internal equilibrium.

For

$$
A=\left(\gamma^{(\alpha)}, g^{(\alpha)}\right)
$$

and $\dot{\gamma}^{(\alpha)}=\Gamma^{(\alpha)}(\sigma)$

$$
\dot{g}^{(\alpha)}=G^{(\alpha)}(\sigma) \equiv \sum_{\beta=1}^{n} h_{\alpha \beta} \dot{\gamma}^{(\beta)} \text {, }
$$

the internal equilibrium state of the crystal

$$
\sigma^{*}=\left(\underset{\sim}{E}\left(t ;, \theta(t), \underset{\#}{\gamma_{\#}^{(\alpha)}}, g_{\#}^{(\alpha)}\right)\right.
$$

is sustained if and only if

$$
\begin{aligned}
& \dot{\gamma}^{(\alpha)}=0 \quad \Rightarrow \dot{g}^{(\alpha)}=0, \\
& \Gamma^{(\alpha)}\left(\sigma^{*}\right)=0 \Rightarrow G^{(\alpha)}\left(\sigma^{*}\right)=0 .
\end{aligned}
$$

If the crystal is in the internal equilibrium state there is no change of the pattern of internal rearrangement.

The condition $(2.97)$ leads to

$$
\hat{i}\left(\sigma^{*}\right)=0 \text {. }
$$


If the crystal is in the internal equilibrium there is no internal dissipation.

For the mechanisms (i) - (ii) the internal equilibrium is attained provided the resolved shear stress vanishes for all $\alpha$ slip system, i.e.

$$
\tau^{(\alpha)}=0 \text { for all } \alpha \text {. }
$$

For the mechanisms (iii) - (vii) the internal equilibrium can be reached if

$$
\tau^{(\alpha)}<g^{(\alpha)} \text { for all slip systems } \alpha
$$

and

$$
\tau^{(\beta)}<g^{(\beta)} \text { for } \beta \neq \alpha
$$

We have

$$
T(t)=\hat{T}\left(E(t), \theta(t), \gamma_{\#}^{(\alpha)}, g_{\#}^{(\alpha)}\right),
$$

i.e. the pure elastic response of the crystal.

3. Hardening effects.

3.1. Theoretical assumptions. To describe hardening effects in crystal the second group of the internal state variables $g^{(\alpha)}$ has been introduced. In previous section we assumed the fundamental hardening law in the form

$$
\dot{g}^{(\alpha)}=\sum_{\beta=1}^{\mathrm{n}} \mathrm{h}_{\alpha \beta}(\theta, \gamma) \dot{\gamma}^{(\beta)} \text { if } \dot{\gamma}^{(\alpha)}>0 \text {, }
$$

where $h_{\alpha \beta}(\theta, \gamma)$ are the temperature and shear strain dependent slip-plane hardening rates. As has been shown by Asaro[2,3] the diagonal terms represent "self-hardening" on a slip system wheras the off-diagonal terms represent coupled or "latent hardening".

3.2. Latent hardening. To describe coupled hardening let us assume

where

$$
h_{\alpha \beta}=h_{1}+\left(h-h_{1}\right) \delta_{\alpha \beta},
$$

where

$$
h_{1}=q \quad \text { and } q \in[1,1.4],
$$

So, we have

$$
h_{\alpha \beta}=q h+h(1-q) \delta_{\alpha \beta},
$$

and for $h$ it is postulated

$$
h(\theta, \gamma)=h_{0}(\theta) \sec h^{2}\left[\frac{h_{0}(\theta) \gamma}{\tau_{S}(\theta)-\tau_{0}(\theta)}\right],
$$

where $\tau(\theta)=g^{(\alpha)}(0)$ for each slip system $\alpha$ is the Critical resolved shear.stress on the crystal slip system, $\tau_{S}=\tau_{S}(\theta)$ represents a temperature dependent saturation value of the flow stress.

$$
\text { It is noteworthy that the relation (3.5) }
$$
is implied by a single slip law of the form

$$
\tau(\gamma, \theta)=\tau_{0}(\theta)+\left[\tau_{S}(\theta)-\right.
$$

$$
\left.-\tau_{0}(\theta)\right] \tan h\left[\frac{h_{0}(\theta) \gamma}{\tau_{s}(\theta)-\tau_{0}(\theta)}\right] \text {. }
$$

3.3. Thermal softening. To describe thermal softening effect observed experimentally it is postulated

$$
h_{0}(\theta)=h_{0}\left[1-\overline{h_{0}}\left(\frac{\theta-\theta_{0}}{\theta_{0}}\right)^{\mu}\right],
$$

where constants $h_{\circ}, \overline{h_{0}}$ and $\mu$ have to be determined basing on experimental results. Similarly we have to identified the material functions $\tau_{S}(\theta)$ and $\tau_{0}(\theta)$.

4. Rate type formulation

4.1. General considerations. As we assumed in Section 2.2 the crystal elasticity is unaffected by slip, then (cf. Hill and Rice[26]) the elastic law have the form

$$
\underset{\sim}{\nabla}{ }^{*}=\underset{\sim}{L}:{\underset{\sim}{D}}^{*},
$$

where $L$ is the tensor of elastic moduli, $\tau$ is the Zaremba-Jaumann rate of Kirchhoff stress. It has been shown in Sec.2.2 that $L$ is derivable from a potential (free energy) function so that it possesses symmetry. We have

$$
\stackrel{\sim}{\tau}^{\nabla}=\underset{\sim}{\dot{\tau}}-{\underset{\sim}{\Omega}}^{*} \cdot \underset{\sim}{\tau}+\underset{\sim}{\tau} \cdot \underset{\sim}{\Omega} *,
$$

where $\dot{\tau}$ is the material rate of Kirchhoff stress. On the other hand

$$
\underset{\sim}{\tau}=\underset{\sim}{\dot{\tau}}-\underset{\sim}{\Omega} \cdot \underset{\sim}{\tau}+\underset{\sim}{\Omega} \text {. }
$$

So, we have

$$
\begin{aligned}
& {\underset{\sim}{\tau}}^{\nabla *}-\underset{\sim}{\nabla}=\sum_{\alpha=1}^{n} \underset{\sim}{\beta}{ }^{(\alpha)} \dot{\gamma}^{(\alpha)}, \\
& {\underset{\sim}{\beta}}^{(\alpha)}={\underset{\sim}{W}}^{(\alpha)} \cdot \underset{\sim}{\tau}-\underset{\sim}{\tau} \cdot \underset{\sim}{W}(\alpha) .
\end{aligned}
$$

where

4.2. Constitutive relations. As a result of previous considerations we obtain the constitutive law as follows

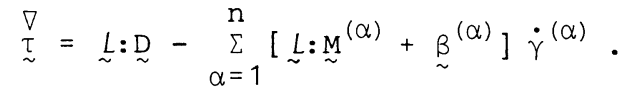

of course, the relation (4.6) has the form of the rate type fundamental evolution equation for the Kifchhoff stress $\underset{\sim}{\tau}$ provided the shearings $\gamma(\alpha)$ can be treated as the internal state variables and may be specified by the evolution equations (the kinetic laws) as the functions of the intrinsic state. In the case of the rate type formulation the intrinsic state is determined by the stress tensor, and a set of the internal state variables which are assumed again as

$$
A(t)=\left(\gamma^{(\alpha)}(t), g^{(\alpha)}(t)\right),
$$

and

$$
\begin{aligned}
& \dot{\gamma}^{(\alpha)}(t)=\Gamma^{(\alpha)}(\sigma), \quad \gamma^{(\alpha)}(0)=\gamma_{0}^{(\alpha)}, \\
& \dot{g}^{(\alpha)}(t)=G^{(\alpha)}(\sigma), \quad g^{(\alpha)}(0)=g_{0}^{(\alpha)},
\end{aligned}
$$

with

$$
\sigma=(\underset{\sim}{\tau}(t), A(t)) .
$$

4.3. Comparison with the internal state variable structure.

Under conditions specified in the previous section we can prove the equivalence between the internal state variable struc- 
ture and the rate type formulation of the constitutive relations for a crystal.

Two material structures developed in Sections 2.3 and 4.2 are materially isomorphic if two conditions are satisfied (cf. Perzyna [55]) :

1. The functional $F$ (defined by (2.22)) which determines the pattern of internal rearrangement in the crystal has to be the same for both structure.

2 . The flux of the Kirchhoff stress tensor has to be chosen properly in the rate type formulation.

5. Description of polycrystalline solids 5.1. Internal state variable constitutive structure. In the description of crystal we introduced a set of the internal state variables as follows

$$
A(t)=\left(\gamma^{(\alpha)}, g^{(\alpha)}\right)
$$

and we postulated

$$
\begin{aligned}
& \dot{\gamma}^{(\alpha)}=\Gamma^{(\alpha)}(\sigma), \quad \gamma^{(\alpha)}(0)=\gamma_{O}^{(\alpha)}, \\
& \dot{g}^{(\alpha)}=G^{(\alpha)}(\sigma), \quad g^{(\alpha)}(0)=g_{O}^{(\alpha)},
\end{aligned}
$$

where

$$
\sigma=(P(t), A(t)) \text {. }
$$

In model proposed the shearings $\gamma^{(\alpha)}$ described the rate of plastic strain tensor $\underset{\sim}{\dot{\mathrm{E}}} \mathrm{P}$ and the rate of the plastic spin $\dot{\sim}^{\mathrm{p}}$ by the relations

$$
\begin{aligned}
& \underset{\sim}{\dot{E}^{\mathrm{P}}}=\sum_{\alpha=1}^{\mathrm{n}} \underset{\sim}{\mu}{ }^{(\alpha)} \dot{\gamma}^{(\alpha)}, \quad \underset{\sim}{\mu}(\alpha)=\underset{\sim}{\mathrm{F}} \cdot \underset{\sim}{\mathrm{M}}{ }^{(\alpha)} \cdot \underset{\sim}{\mathrm{F}},
\end{aligned}
$$

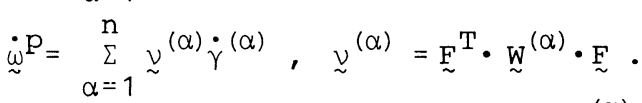

The critical shear stresses $g^{(\alpha)}$ described the hardening-softening effects and latent hardening.

To describe all these effects in macroscopic-phenomenological theory of polycrystalline solids we have to introduce such conceptions as isotropic hardening and kinematic hardening.

This reasoning suggests two internal state variables $k$ and $\alpha$. The first describes isotropic harden ing and the second the kinematic hardening and is interpreted as the residual stress.

So, we have a set of the internal state variables

$$
A(t)=\left(\underset{\sim}{E}{ }^{p}, \underset{\sim}{\omega}, k, \underset{\sim}{\alpha}\right)
$$

and the evolution equations as follows

$$
\begin{aligned}
& {\underset{\sim}{\dot{E}}}^{\mathrm{p}}(t)=\underset{\sim}{\hat{E}}(\sigma), \quad \underset{\sim}{\mathrm{E}} \mathrm{P}(0)={\underset{\sim}{\mathrm{E}}}_{\mathrm{P}}^{\mathrm{p}}, \\
& \dot{\sim}^{\mathrm{p}}(t)=\hat{\Omega}(\sigma), \quad{\underset{\sim}{\omega}}^{\mathrm{p}}(0)={\underset{\sim}{\omega}}_{0}^{\mathrm{p}}, \\
& \dot{K}(t)=\hat{K}(\sigma), \quad k(0)=k_{0}, \\
& \underset{\sim}{\dot{\alpha}}(t)=\hat{\sim}(\sigma), \quad \underset{\sim}{\alpha}(0)={\underset{\sim}{\alpha}}_{0},
\end{aligned}
$$

where

$$
\sigma=(E(t), \theta(t), A(t)) .
$$

The internal dissipation is given by

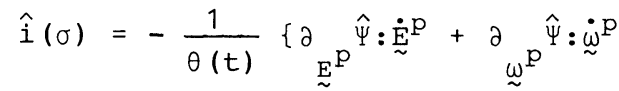

$$
\begin{aligned}
& +\partial_{K} \hat{\psi} \dot{\dot{K}}+\partial_{\underset{\sim}{\alpha}}^{\hat{\psi}: \underset{\sim}{\dot{\alpha}}\}} \text {. }
\end{aligned}
$$

Let us discuss the second term in the expression (5.8). This is contribution to the rate of the internal dissipation given by the plastic spin ${\underset{\sim}{P}}^{P}$.

We define

$$
\partial \underset{\omega}{p} \hat{\Psi}={\underset{\sim}{T}}_{M}, \quad{\underset{\sim}{M}}_{M}^{T} \neq \underset{\sim}{T}{\underset{\sim}{M}}_{\sim}^{T}
$$

and inter̃pret $T$ as the couple stress tensor. If in the $\tilde{\tilde{M}}^{\mathrm{M}}$ theory developed we postulate

$$
\partial \underset{\omega^{p}}{p} \hat{\Psi}=0 \text {, }
$$

there is no coupĨe stress tensor, then the plastic spin can not play the role of the internal state variable. Then (cf.Mandel [45] and Perzyna [57])

$$
A(t)=\left(\underset{\sim}{E^{P}}, K, \underset{\sim}{\alpha}\right) \text {. }
$$

This is almost natural generalization to the description of the polycrystalline aggregate.

The other proposition had been presented by Perzyna [54]

$$
A(t) \equiv\left\{{\underset{\sim}{E}}^{p}(t), K(t), \underset{\sim}{\Gamma}(k)(t)\right\},
$$

where $\Gamma_{\sim}^{(k)}$ denots a set of the dislocation density tensors. The dislocation arrangement tensors $\Gamma(\mathrm{k})$ were first introduced into the theor̃y of plasticity for the description of the structural rearrangement by E.Kroner $[34,35,36]$. These tensors are interpreted as the excess dislocation density $\Gamma^{(1)}$ the excess dislocation loop density $\Gamma_{\sim}(2)$, the excess dislocation pair loop deñsity $\Gamma^{(3)}$ etc. One could continue in this manner and get the infinite and complete set of internal state variables which would describe any detail of the dislocation rearrangement. Since it is not likely that all the particulars on a microscopic scale are important for the macroscopic viscoplastic behaviour of the polycrystalline aggregate, but rather that certain averages only are macroscopically effective and important for phenomenological description, one is inclined to introduce a finite set of the internal state variables $\Gamma(k)$.

The pheñomenological and macroscopic structure-parameter formulation presented by J.R.Rice [66] is also a good example of such description. He introduced

$$
A(t) \equiv\left\{{\underset{\sim}{E}}^{\mathrm{P}}(t), \rho(t), \underset{\sim}{\lambda}(t)\right\}
$$

and interpreted $\rho$ as the net dislocation density, or some average measure of the kind $\mathrm{b} / \mathrm{L}$, where $\mathrm{b}$ is the Burgers vector and $\mathrm{L}$ is a distance between strong dislocation pinning points, or instead just as the flow stress at some fixed temperature, sufficiently low that creep effects are absent; and $\lambda$ as a measure of the anisotropy that has beẽn induced by plastic deformation. It is noteworthy that such parameter characterization would seem suitable so long as the deformat- 
ion is not so large as to induce significant preferred orientation and texturing.

Returning to a set of the internal state variables (5.5) let us formulate the evolution equations based on the generalization of the viscoplastic flow mechanism discussed in section 2.5.

We postulate for temperature and rate dependent response

$$
\begin{aligned}
& \dot{\sim}^{\mathrm{E}}(t)=n\left\langle\Phi\left[\frac{\tilde{f}(\cdot)}{k}-1\right]\right\rangle \partial_{\underset{\sim}{T}} \tilde{f}, \\
& \underset{\sim}{\alpha}(t)=p(\kappa, \theta)\left[{\underset{\sim}{\alpha}}_{\text {sat }}-\underset{\sim}{\alpha}\right]\left[{\underset{\sim}{\dot{\mathrm{E}}}}^{\mathrm{p}}:{\underset{\sim}{\dot{\mathrm{E}}}}^{\mathrm{p}}\right]^{\frac{1}{2}}, \\
& \underset{\sim}{\dot{K}}(t)=h_{1}(\kappa, \theta)\left[{\underset{\sim}{\dot{E}}}^{p}:{\underset{\sim}{\dot{\mathrm{E}}}}^{\mathrm{p}}\right]^{\frac{1}{2}}+\mathrm{h}_{2}(\kappa, \theta) \underset{\sim}{\alpha}:{\underset{\sim}{\dot{\mathrm{E}}}}^{\mathrm{p}} \\
& -\mathrm{h}_{3}(\kappa, \theta) \text {, }
\end{aligned}
$$

where $\tilde{f}(\cdot)=\tilde{f}\left(\tilde{j}_{1}, \tilde{j}_{2}^{\prime}, \tilde{j}_{3}^{\prime}\right)$, of lower yield stress denote invariants of the stress tensor,

$$
\underset{\sim}{\tilde{T}}=\underset{\sim}{T}-\underset{\sim}{\alpha}
$$

and $\mathrm{p}_{1} \mathrm{~h}_{1}, \mathrm{~h}_{2}$ and $\mathrm{h}_{3}$ are the material functions

$$
n \rightarrow \infty
$$

then from

$$
\tilde{f}(\cdot)=K\left\{1+\Phi^{-1}\left[\frac{\left(\dot{\mathrm{E}}^{\mathrm{p}}: \dot{\mathrm{E}}^{\mathrm{p}}\right)^{\frac{1}{2}}}{\eta}\left(\partial_{\mathrm{T}} \tilde{f}: \partial_{\sim} \tilde{\sim}\right)^{-\frac{1}{2}}\right]\right\}
$$

we can deduce that

$$
\tilde{f}(\cdot)=k
$$

and

$$
\dot{\mathrm{E}}^{\mathrm{p}}(t)=\Lambda \partial_{\mathrm{T}} \tilde{f} .
$$

Additionaly we have to pur

$$
\mathrm{h}_{3}=0 \text {. }
$$

Equations $(5.14),(5.14)$ with $(5.20),(5.15)$ $(5.18)$ and $(5.19)^{\prime}$ describe inviscid elasto-plastic response of polycrystalline solids.

\section{References}

1. Argon, A.S., ed.,Constitutive Equations in Plasticity, MIT Press, Cambridge, Mass. , 1975

2. Asaro, R.J., Advances in Applied Mechanics, vol.23, Academic Press

3. Asaro,R.J., A.Appl.Mech. ,50,921-934, 1983

4. Asaro, R.J., and Needleman, A., Acta Metall.,33, 923-953,1985.

5. Asaro, R.J. and Rice,J.R. ,J.Mech.Phys. Solids, 25, 309-338, 1977 .

6. Ashby,M.F. and Frost,H.J., in Constitutive Equation in Plasticity (ed.A.S.Argon) MIT Press, Cambridge, Mass. 1975

7. Bingham, E.C., Fluidity and Plasticity, McGraw--Hill, New York 1922
8. Bishop, J.F.W. and Hill, R., Phil.Mag., $42,414-427,1951$

9. Bishop,J.F.W., and Hill,.., Phil.Mag., $42,1298-, 1951$

10. Budiansky, B. and Wu, T.Y., Proc. 4 th Congress Appl.Mech.,p.1175,1962

11. Bui,H.D., Zaoui,A. and Zarka,J., in Foundations of Plasticity (ed.A.Sawczuk) Proc.Int.Symp.1972, Noordhoff, Leyden 1973, pp. 51-75

12. Campbell,J.D., Material Sci.Engng.,12, 3-21, 1973

13. Campbell,J.D. and Ferguson,W.G. ,Phil. Mag. , 81, 63-82, 1970

14. Evans, A.G. and Rawlings, R.D. ,Phys. Stat. Sol. $34,9-31,1969$

15. Gilman,J.J., J.Appl. Phys. ,36, 2772-2777, 1965

16. Gilman,J.J., Micromechanics of flow in solids,McGraw-Hill, New York 1969

17. Gilman,J.J. and Johnston,W.G. ,Solids State Physics, 13,147,1962

18. Hauser,F.E.,Simmons, J.A. and Dorn, J.E., in Response of Metals to High Velocity Deformation, Wiley, New York 1961,pp.93$-114$

19. Havner,K.S., Proc.Roy.Soc. $378,1981,329$

20. Havner.K.S. and Shalaby,A.H.,Proc.R.Soc. A358, 47-7-, 1977

21. Hill,R., J.Mech. Phys.Solids, 14, 95-102, 1966

22. Hill,R., J.Mech.Phys.Solids, 15, 79-95, 1977

23. Hill, R. ,Proc.R. Soc., Lond. A326, 131-147, 1972

24. Hill, R., Math.Proc.Camb. Phil.Soc.,85, $179-191,1979$

25. Hill, R. and Havner, K.S., J.Mech. Phys.Solids, 30, 5-22, 1982

26. Hill. R. and Rice, J.R., J.Mech. Phys. Solids, $20,401-413,1972$

27. Hohenemser,K. and Prager,W., ZAMP, 12, $216-226,1932$

28. Hutchinson, J.W. ,Proc.R. Soc.Lond.A, 319 , 247,1970

29. Hutchinson, J.W., Proc,R.Soc.Lond.A, 348, $101-127,1976$

30. Iwakuma,T. and Nemat-Nasser, S.,Proc.R. Soc. Lond. A, 394, 87-119, 1984

31. Kocks,U.F., Met.Trans.,1, 1121,1970

32. Kocks,U.F., in Constitutive Equations in Plasticity (ed.A.S.Argon) , 81-115, 1975

33. Kocks,U.F., Argon, A.S. and Ashby,M.F., Thermodynamics and Kinetics of Slip, Pergamon Press, 1975

34. Kroner, E. ,Arch. Ration. Mech. Ana 1., , 4,273$-334,1960$

35. Kröner, E., J.Math. Phys. ,42, 27-37, 1962

36. Kroner,E., in Theory of Crystal Defects, (ed.B.Gruber), Academic Press, New York, $231-256,1966$

37. Kumar,A.,Hauser,F.E. and Dorn, J.E., Acta Metalurgica, 16, 1189-1191,1968

38. Kumar,A. and Kumble,R.G., J.Applied Physics, 40,3475-3480,1969

39. Lee,E.H. ,J.Appl.Mech., 35, 1-6, 1969

40. Lindholm, U.S., J.Mech. Phys.Solids, 12, $317-335,1964$

41. Lindholm,U.S., in Behaviour of Materials under Dynamic Loading, ASME, 42-61,1965

42. Lindholm,U.S., in Mechanical Behaviour of Materials under Dynamic Loads, (ed. U.S.Lindholm), Springer-Verlag,77-95, 1968

43. Malvern,L.E.,J,Appl. Mech.,18,203-208, 1951 
44. Mandel,J., in Problems of Plasticity, (ed.A.Sawczuk), Noordhoff Int.Publishing, 135-143,1972

45. Mandel,J., Foundation of Continuum Thermodynamics, (ed.J.J.Domingos,M.N.R. Nina, and J.H.Whitelaw), 283-304

46. Milstein,F. and Hill, R., J.Mech. Phys., $25,457-477,1977$; 26,213-239,1978; 27, $\frac{25}{25}-279,1979$

47. Mason,W.P., J.Acoustical Soc.Amer., 32 , $458-472,1960$

48. Mason,W.P., J.Appl.Phys .,35, 2779-2781, 1964

49. Nabarro,F.R.N., Theory of Crystal Dislocations, Oxford Univ.Press, 1967

50. Needleman,A., Asaro,R.J., Lemonds, J., Peirce, D., Comp.Meth.Appl.Mech., Eng . , $52,689-708,1985$

51. $\overline{P a n}, \mathrm{~J}$. and Rice,J.R., Int.J.Solids Struct. $, 19,973,1983$

52. Perzyna, P., Quarterly Appl.Math., 20. $321-332,1963$

53. Perzyna,P., Advan.Appl. Mech . ,9, 243-377, 1966

54. Perzyna,P., Advan.Appl.Mech., 11,313$-353,1971$

55. Perzyna,P., Arch.Mech., 27,473-484, 1975

56. Perzyna,P., Arch.Mech. , $\underline{29}, 607-624,1977$

57. Perzyna, P., in Recent Developments in Thermomechanics of Solids, (ed.G.Lebon and P.Perzyna), Springer-Verlag,95-220, 1980

58. Perzyna,P., Arch.Mech., 32,403-420,1980

59. Perzyna, P. and Pecherski, R.B., Arch.Mech, $35,423-436,1983$

60. Peirce,D. Asaro,R.J. and Needleman,A., Acta Metall., 30,1087-1119,1982
61. Peirce,D., Asaro, R.J. and Needleman,A., Acta Metall., 31,1951-1976,1983

62. Prager,W., Mecanique des solids isotropes au del du domain elastique. Memorial Sci., Math., Paris 1937, 87

63. Randal,M.R.D. and Campbeli, J.D., University of Oxford, Report $\mathrm{N}^{\circ} 1047 / 72$

64. Rice,J.R., J.Appl.Mech.,37, 728-737,1970

65. Rice,J.R., J.Mech.Phys.Solids, 19,433$-455,1971$

66. Rice,J.R., in Constitutive Equations in Plasticity (ed.A.S.Argon), MIT Press, 1975 , pp. 23-80

67. Seeger,A., Phil.Mag., 46,1194-1217,1955

68. Seeger,A., in Handbuch der Physik VII/2 (ed.S.Flügge), Springer 1958

69. Shindoh,A., Kobe University, Private communication, 1986

70. Shioiri,J., Satoh,K. and Nishimura,K. in High Velocity Deformation in Solids, IUTAM Symp.Proc. (ed.K. Kawata and J.Shioiri), Springer 1979, pp.50-66

71. Sokolowski,V.V., Doklady Akad.Nauk SSSR, 60,775-778,1325-1328, 1948

72. Stein, D.F. and Low, J.R., Jr., J.Appl. Phys., 31,362-369, 1960

73. Taylor, G.I., J.Inst.Metals, 62, 307-324, 1938

74. Teodosiu,C. and Sidoroff,F., Int.J.Eng. Sci., 14,165-176,1976

75. Kröner $\overline{, E}$. and Teodosiu, C., in Problems of Plasticity, Int.Symp.Proc. (ed. A.Sawczuk), Noordhoff, Leyden 1973, pp. 45-88

76. Zarka,J., J.Mech.Phys.Solids, 20,179$-195,1972$ 